\title{
DOE/ER/13867-15
}

\section{THEORETICAL STUDIES OF ATOMIC TRANSITIONS}

Renewal Progress Report

Period: April 1, 1994 - March 31, 1997

Charlotte FROESE FISCHER

Professor of Computer Science, Mathematics, Chemistry, and Physics

Box 16796, Vanderbilt University

Nashville, Tennessee 37235 USA

Phone: (615) 322-2926; email: cffvuse.vanderbilt.edu

http://www . vuse.vanderbilt.edu/"cff/cff.html

DISTRIBUTION OF THIS DOCUMENT IS UNLIMTED h

MASTER

July, 1996

Prepared for the U.S. Department of Energy under grant number DE-FG05-88ER13867 


\section{DISCLAMMER}

This report was prepared as an account of work sponsored by an agency of the United States Government. Neither the United States Government nor any agency thereof, nor any of their employees, makes any warranty, express or implied, or assumes any legal liability or responsibility for the accuracy, completeness, or usefulness of any information, apparatus, product, or process disclosed, or represents that its use would not infringe privately owned rights. Reference herein to any specific commercial product, process, or service by trade name, trademark, manufacturer, or otherwise does not necessarily constitute or imply its endorsement, recommendation, or favoring by the United States Government or any agency thereof. The views and opinions of authors expressed herein do not necessarily state or reflect those of the United States Government or any agency thereof. 


\section{DISCLAIMER}

Portions of this document may be illegible electronic image products. Images are produced from the best available original document. 


\section{Research Progress Report}

With the aid of powerful workstations and our software modified for large-scale computation, considerable progress has been made during the last few years. This progress is in the form of increased accuracy for transition probablity studies along with estimates of uncertainty, the study of nuclear effects on atomic properties such as hyperfine interactions and isotope shifts, the study of relativistic correlation in some large and small systems, and the extension of spline methods to regions of higher energy where multiple continuum channels are present.

A number of research groups have collaborated in this effort. A project, referred to as $S A M$, for Systematic, Accurate, Multiconfiguration studies of atomic properties currently consists of Dr. M.R. Godefroid and his group at the Free University of Brussels, Tomas Brage, a former Postdoc now at NASA, Goddard, and Per Jönsson, a former student from Lund, now a Postdoc on the grant. Alan Hibbert and some of his associates at Belfast have also joined the group. More information about the group can be obtain from the World Wide Web (URL: http://aniara.gsfc.nasa.gov/sam/sam.html) where results are being collected as they are submitted for publication. The appendix lists grant related papers that appeared since 1994, papers presented at conferences, and the papers submitted. Not mentioned are papers in preparation.

\section{The computational tools}

The Vanderbilt group has concentrated on developing the computational capability for dealing with the large expansions needed for achieving unprecedented accuracy for systems with more than three electrons. In this we were assisted by Dr. Lennart Sturesson of Lund University, now a high-school teacher of physics, who developed the code for generating wave function expansions by applying rules that define a model for correlation in a complex atom. Two packages are used extensivley. The MCHF package starts with the Schrödinger equation and adds relativistic Breit-Pauli corrections, and finite nuclear mass corrections, when needed. This package has been converted to parallel execution on a cluster of workstations using PVM (parallel virtual machine) software and more recently to the standard message passing interface (MPI). The GRASP package starts with the Dirac-Fock form of a similar variational procedure where the Breit and QED corrections are added as needed. A modular version, suitable for calculations about an order of magnitude larger than before, has been developed and now published as GRASP92. Both these codes use the Davidson algorithm [1] developed for finding a few selected eigenvalues and eigenvector of a matrix.

An important breakthrough has been the development of a theory for evaluating matrix elements between configuration states where the orbitals of one are non-orthogonal to the other without first transforming the wavefunction expanded in terms of symmetry adapted configuration states to a wavefunction expanded in determinants. This allows independent optimization of states without concern about subsequent non-orthogonality of orbitals and keeping the efficient configuration state expansions. Our first application is to transition studies but the methodology can also be applied to other properties. This methodology is an extension of the theory first developed by the Quantum Chemistry group at Lund, for expansions in terms of determinants. M.R. Godefroid and his group are looking into the application of this technique to Breit-Pauli calculations in order that interactions can be computed from independently optimized LS states. Per Jönsson, presently visiting the Quantum Chemistry Department at Lund, is investigating the extension of this approach to the relativistic case, of $j-j$ coupled symmetry adapted functions.

Through an NSF Physics infrastructure grant, the group has access to a workstation farm consisting of 14 DEC Alpha systems. The group also has entered into a Joint Study Agreement with IBM providing limited access to an SP-2 system. Thus experience has been gained on three platforms for distributed parallel computing. 


\section{Research Highlights}

The titles of the journal publications listed in the Appendix indicate the diverse properties and atomic systems - large and small, non-relativistic and relativistic - that have been studied. Here we wish to draw attention to only the major achievements.

\subsection{The long-standing $\mathrm{Li}$ and $\mathrm{Na}$ discrepancy}

The year 1995 has seen the resolution of the long-standing discrepancy between theory and experiment for the oscillator strengths of the resonance line of both $\mathrm{Li}$ and $\mathrm{Na}$. In 1982 some beam laser measurements were reported with extremely small error bars, though the values differed from nearly all theory. For a relatively simple system like lithium, this discrepancy was disturbing. At the 5th International Colloquium on Atomic Spectra and Oscillator Strengths for Astrophysical and Laboratory Plasmas (Paris, France, 28-31 August, 1995) we reported accurate length and velocity oscillator strengths for $\mathrm{Li}$ of 1.49391 and 1.49374 , respectively, in excellent agreement with a new experimental value of 1.4934(32) reported by Volz and Schmoranzer [2]. The length value is in perfect agreement to 5 decimal places with the Yan and Drake [3] Hylleraas value where length and velocity are in agreement to 6-decimal places.

Also reported at the same meeting was a new value of the multiplet strength for sodium of 37.26 au. to be compared with three new experimental values of of 37.26, (Volz et al [4]), 37.24 (Richling et al [5]), and 37.30 (Oates et al [6]). Details and further references for both transitions may be found in Godefroid et al [7]. Unlike earlier efforts, the sodium calculation included core-polarization and core-core calculations more rigorously, the largest calculations being performed on an IBM SP2 through a Joint Study Agreement with IBM. The quality of the wave function was evaluated by using the same function for the determination of accurately known hyperfine parameters which probe the quality of the wave function near the nucleas. The lithium calculation was a further test of our methodology since this is not a system where configuration methods can compete with the more powerful Hylleraas method, but our calculation showed that accuracy to four decimal places could be achieved.

Unlike lithium, at a high level of accuracy, a non-relativistic line strength for $\mathrm{Na}$ is in error already in the third significant digit. The finite nucleas and relativistic effects were determined from smaller GRASP calculations and compared with MCHF results. Both methodologies were needed for bringing results into agreement with the three new experiments, all in essential agreement but differing substantially from the earlier Gaupp et al [8] measurement with extremely small error bars.

\subsection{Isotope Shift}

The $1362 \AA$ resonance line of B II has great astrophysical interest in that the the ${ }^{10,11} B$ isotopic ratios may provide information about production processes of light elements in interstellar media. The line has been observed in the spectra of $\delta$ Sco acquired with the Goddard High Resolution Spectrograph aboard the Hubble Space Telescope. An isotope shift or $13.3 \mathrm{~m} \AA$ was predicted by our computations, a value that has now been confirmed. Analysis of the observed data yielded an isotope shift of $13.7 \pm 3.5 \mathrm{~m} \AA$ [9].

\subsection{Intercombination Lines}

In an early study of the $2 s^{2}{ }^{1} S_{0}-2 s 2 p^{3} P_{1}$ intercombination line of C III [10], a systematic procedure was used to compute properties within different models - outer correlation, core-polarization, partial core-core correlation - that made it possible to predict the transition rate and, by monitoring both 
the trends within a model as the size of the wave function expansion increased as well as the effect of the model, a final recommended value could be reported along with an uncertainty estimate. According to $E$. Träbert, our value for the transition rate of $103 \pm 3 \mathrm{~s}^{-1}$ is in perfect agreement with the result his group plans to report at the Zeeman Internation Conference on Atomic Physics in Amsterdam, August, 1996. Their result is said to be close to $103 \mathrm{~s}^{-1}$ with an error of $1 \%$.

A similar methodology has been used extensively for other atoms [11]. Since the transition arises solely from a spin-orbit interaction, the iso-electronic sequence was also investigated using GRASP where calculations for atoms as large as $\mathrm{U}^{+89}$ were performed. For the light atoms, the MCHF calculations appeared to be the more accurate.

For the $2 s^{2}{ }^{1} S_{0}-2 s 3 p^{1,3} P_{1}$ calculations, the new non-orthogonality methodology has been used and applied to an iso-electronic sequence. The computed results are in better agreement with semi-empirical trend predicted by Curtis et al [12], than the original experimental data.

\subsection{Photoionization in Negative Ions}

The ability of MCHF to accurately represent the localized charge distribution or perturber in the continuum of a negative ion was exploited in the study of photodetachment from the $1 s 2 s 2 p{ }^{4} P^{\circ}$ state in negative helium [13]. The position of the $1 s 2 p^{2}{ }^{4} P$ resonance was predicted in agreement with experiment to experimental accuracy [14] though the width estimate varied somewhat, depending on the assumption about the cross-section of the background process. Photon energies of up to $4 \mathrm{eV}$ were considered which covers the whole energy region up to $\mathrm{He}(n=4)$ threshold. A B-spline basis together with inverse iteration was employed to solve the multichannel problem. Several additional Feshbach resonances were found and the angular distribution was studied.

\section{Summary}

This research has been a collaborative effort, particularly with Michel Godefroid, without whose dedication the independently optimised method for transition studies would not have been developed, and with Per Jönsson who developed the hyperfine codes for the MCHF and GRASP92 packages. With the co-operation of Farid Parpia, supported on the grant during 1991-2, the latest GRASP code, modified for large-scale relativistic computation has been published. We expect this release to result in the extension and improvement of the package by scientists not necessarily part of our group. We have also benefited from an initial collaboration with the Theoretical Institute of Physics and Astronomy, Vilnius, Lithuania, for speeding up the angular integrations for the MCHF package. This collaboration is now continuing under an NSF collaboration grant.

\section{References}

[1] A. Stathopoulos and C. Froese Fischer, Computer Phys. Commun. 79, 268 (1994).

[2] U. Volz and A. Schmoranzer, Physica Scripta (in press)

[3] Z-C Yan and G.W.F. Drake, Phys. Rev. A, 52, 3711 (1995).

[4] U. Volz, M. Majerus, H. Liebel, A. Schmoranzer, Phys. Rev. Lett. 76, 286 (1996)

[5] H. Richling, H. Knöckel, and E. Tiemann (private communication) (1995).

[6] C.W. Oates, K.R. Vogel, and J.L. Hall, Phys. Rev. Lett. 76, 2866 (1996).

[7] M.R. Godefroid, C. Froese Fischer, and P. Jönsson, Physica Scripta (in press). 
[8] A. Gaupp, P. Kuske, and J.J. Andrä, Phys. Rev. A, 26, 3351 (1982).

[9] S.R. Federman, D.L. Lambert, J.A. Cardelli, and Y. Sheffer, Nature (submitted) (1996).

[10] C. Froese Fischer, Physica Scripta, , 323 (1994).

[11] J. Fleming, T. Brage, K.L. Bell, N. Vaeck, A. Hibbert, M. Godefroid, and C. Froese Fischer, Ap. J. 455, 758 (1995).

[12] L.J. Curtis, S.Ti Maniak, R.W. Ghrist, R.E. Irving, D.G. Ellis, M. Henderson, M.H. Kacher, E. Träbert, J. Granzow, P. Bengtsson, and L. Engström, Phys, Rev. A 51, 4575 (1995).

[13] J. Xi and C. Froese Fischer, Phys. Rev. A 53, 3169 (1996).

[14] C.W. Walter, J.A. Seifert, and J.R. Peterson, Phys., Rev. A 50, 2257 (1994). 


\section{APPENDIX Publications and Presentations}

\section{A. Journal Publications}

1. C. Froese Fischer, Transition Probabilities in O III, Physica Scripta. 49, 51-61 (1994).

2. C. Froese Fischer, Allowed Transitions and Intercombination Lines in C III and C II, Physica Scripta, 49, 323-330 (1994).

3. T. Brage, C. Froese Fischer, and P. Jonsson, Effects of core-valence and core-core correlation on the line strength of the resonance lines in $\mathrm{Li} I$ and $\mathrm{Na} I$, Phys. Rev. A 49, 2181-2184 (1994).

4. J. Carlsson, P. Jonsson, L. Sturesson, and C. Froese Fischer, Lifetimes and transition probabilities of the boron atom calculated with the active-space multiconfiguration Hartree-Fock method, Phys. Rev. A 49, 3426-3431 (1994).

5. T. Brage and C. Froese Fischer, Spline-Galerkin Calculations for Rydberg Series of Calcium, Physica Scripta, 49 , 651-660 (1994).

6. T. Brage and C. Froese Fischer, Spline-Galerkin methods applied to Rydberg series between the $4 s^{2} S$ and $3 d^{2} D$ limits of calcium, Phys. Rev. A 50, 2937-2947 (1994).

7. C. Froese Fischer, M. Tong, M. Bentley, Z. Shen, and C. Ravimohan, A Distributed-Memory Implementation fo the MCHF Atomic Structure Package, J. of Supercomputing 8 , 117-134 (1994).

8. P. Jonsson, S.G. Johansson, and C. Froese Fischer, Accurate Calculation of the isotope shift and hyperfine structure in the boron (B II) line at 1362 A, Astrophysics J. 429, L45-L48 (1994).

9. C. Froese Fischer and P. Jönsson, MCHF Calculations for Atomic Properties, Computer Phys. Commun. 84, 37-58 (1994).

10. C. Froese Fischer, Lifetime of $2 s 2 p^{2} 3 s^{3} P$ in $N I I$ and other allowed transitions, Z. Phys. D 32, 21-26 (1994).

11. M. Tong, C. Froese Fischer, and L. Sturesson, Systematic Transition Probability Studies for Neutral Nitrogen, J. Phys. B: 27, 4819 (1994).

12. T. Brage and C. Froese Fischer, Spline-Galerkin Methods for Rydberg Series, including BreitPauli Effects, J. Phys. B: J. Phys. B: 27, 5467 (1994).

13. A. Bernotas, C.Froese Fischer, J. Kaniauskas, V. Tutlys, Mixed-valence state treatment for $X$-ray emission spectra of cuprate superconductors, J. Phys. Condensed Matter: 68349 (1994).

14. J. Bieron, C. Froese Fischer, and A. Ynnerman, Note on MCDF correlation calculation fo high-Z ions, J. Phys. B: 27, 4829 (1994).

15. J. Bieron, F.A. Parpia, C. Froese Fischer, and P. Jönsson, Large-scale multi-configuration Dirac-Fock calculations of hyperfine interaction constants for the nd(2) levels of Sc II and Y II, Phys. Rev. A 51, 4603 (1995). 
16. A. Aboussaïd, M.R. Godefroid, P. Jönsson, and C. Froese Fischer, MCHF calculations of hyperfine-induced transitions in helium-like ions, Phys. Rev. A 51, 2031 (1995).

17. C. Froese Fischer, J.E. Hansen, and H.W. van der Hart, $A b$ initio studies of the $4 s 4 p^{2}{ }^{4} P$ state in $\mathrm{Ca}^{-}$, Phys. Rev. A 51, 1999 (1995).

18. A. Ynnerman and C. Froese Fischer, $M C D F$ calculation of the $2 s^{1} S_{0}-2 s 2 p{ }^{3} P_{1}$ spin-forbidden transition for the Be-like iso-electronic sequence, Phys. Rev. A 51, 2020 (1994).

19. J. Olsen, M.R. Godefroid, P. Jönsson, P.A. Malmqvist, and C. Froese Fischer, On the use of RAS configuration interaction expansions to deal with non-orthogonalities in atomic transition probability calculations, Phys. Rev. E 52, 4499 (1995).

20. C. Froese Fischer and T. Brage, Splines in Atomic Structure Calculations, Proceedings of the Atomic Collisions Symposium in Honor of Christopher Bottcher, AIP Press, 1995.

21. A. Ynnerman and C. Froese Fischer, Allowed Transitions and Intercombination lines in $B$ II, Z. Phys. D: 34, 1 (1995).

22. C. Froese Fischer, A. Ynnerman and G. Gaigalas, MCHF calculations for the electron affinity of Boron, Phys. Rev. A 514611 (1995).

Ap. J. 450, 473-476 (1995).

23. M. Godefroid, J. Olsen, P. Jönsson, and C. Froese Fischer, Accurate MCHF calculations of oscillator strengths in light atoms: the $B$ II line at $1361 \AA$ Ap. J. 450, 473 (1995).

24. J. Carlsson, P. Jönsson, M. Godefroid, and C. Froese Fischer, Acccurate multiconfiguration Hartree-Fock calculations of isotope shifts in CIV and C I, J. Phys. B: 283729 (1995).

25. T. Brage, C. Froese Fischer, and P. Judge, Transition probabilities for the UV0.01 Multiplet in $N$ III, Astophys. J. 445, 457 (1995).

26. P. Jönsson, C. Froese Fischer, and J. Bieron, MCHF calculations for low-lying excited ${ }^{2} S$ states in Lithium, Phys. Rev. A 52, 4262 (1995).

27. J. Fleming, T. Brage, K.L. Bell, N. Vaeck, A. Hibbert, M.R. Godefroid, and C. Froese Fischer. Systematic studies of N IV transitions of astophysical importance Ap. J, 455, 758-768 (1995).

28. A. Stathopoulos; A. Ynnerman, and C. Froese Fischer, A PVM Implementation of the MCHF Atomic Structure Package, Int'l J. Supercomputing Applications and High-Performance Computing 10, 41-61 (1996).

29. T. Brage, D. Leckron, and C. Froese Fischer, Core-valence and core-core correlation effects on hyperfine-structure parametersand oscillator strengths in Tl II and TI III Phys. Rev. A 53, 192-200 (1996).

30. J. Bieron, P. Jönsson, and C. Froese Fischer, Large-scale multiconfiguration Dirac-Fock calculations of the hyperfine-structure constants of the $2 s^{2} S_{1 / 2}, 2 p^{2} P_{1 / 2}$, and $2 p^{2} P_{3 / 2}$ states of lithium, Phys. Rev. A 53, 2181-2188 (1996).

31. F.A. Parpia, C. Froese Fischer, and I.P. Grant, GRASP92: A package for large-scale relativistic atomic structure calculations, Comput. Phys. Commun. 94, 249-271 (1996).

32. J. Xi and C. Froese Fischer, Cross section and angular distribution for the photodetachment of $\mathrm{He}^{-}\left(1 s 2 s 2 p^{4} \mathrm{P}^{\circ}\right)$ below the $n=4$ threshold, Phys. Rev. A 53, 3169 (1996). 
33. C. Froese Fischer and G. Gaigalas, The electron affinity of $2 s 2 p^{2}{ }^{4} P$ in boron, J. Phys. B: At. Mol. Opt. Phys. 29, 1169-73 (1996).

34. P. Jönsson, A. Ynnerman, C. Froese Fischer, M.R. Godefroid, and J. Olsen, Large-scale $M C H F$ and $C I$ calculations of the transition probability and hyperfine structures in the sodium resonance transition, Phys. Rev. A 53, 4021 (1996).

\section{B. Technical Reports, Conference Presentations, Invited Talks}

1. P. Jonsson and C. Froese Fischer, MCHF calculations of isotope shifts; I Program implementation and test runs; II Large-scale active space calculations, Technical Report DE-FG0588ER13867, Department of Computer Science, Vanderbilt University, 1994.

2. J. Bieron, P. Jönsson, F.A. Parpia, C. Froese Fischer, Hyperfine studies from large scale relativistic configuration-interaction calculations, Bulletin of the APS, 39, 1182 (1994) (poster, DAMOP meeting)

3. T. Brage and C. Froese Fischer, Spline-Galerkin Calculations of Rydberg series above the first ionization limit of Calcium, Bulletin of the APS, 39, 1207 (1994) (poster, DAMOP meeting)

4. J. Carlsson, M.B. Gaarde, P. Jönsson, L. Sturesson, C. Froese Fischer, Active space MCHF calculations of lifetimes and hyperfine structures in the Rydberg series of the boron atom, (poster), 1994 EGAS conference, Barcelona, SPAIN.

5. J. Carlsson, P. Jönsson, and C.Froese Fischer, Systematic studies of isotope shifts in light atoms by active set MCHF calculations, (poster), 1994 EGAS conference, Barcelona, SPAIN.

6. J. Olsen, M. Godefroid, P. Jönsson, P.A. Malmqvist, C. Froese Fischer, On the use fo RAS configuration interaction expansions to deal with non-orthogonalities in transition probability calculations, Abstract of 14th International Conference on Atomic Physics, Boulder, 1994.

7. C. Froese Fischer, J.E. Hansen, H.W. van der Hart, Core polarization effects for negative ions, Abstract, 14th International Conference on Atomic Physics, Boulder, 1994.

8. A. Abouusaïd, M. Godefroid, P. Jönsson, and C. Froese Fischer, MCHF Calculations of Hyperfine-induced transitions in He-like ions, Abstract, 14th International Conference on Atomic Physics, Boulder, 1994.

9. A. Ynnerman and C. Froese Fischer, MCDF calculation of the spin-forbidden transition $2 s^{2}{ }^{1} S_{0}-2 s 2{ }^{3} P_{1}$ for the Be-like isoelectronic sequence, Abstract, 14th International Conference on Atomic Physics, Boulder, 1994.

10. J. Bieron, C. Froese Fischer, Large-scale relativistic calculations of atomic properties, Abstract, 14th International Conference on Atomic Physics, Boulder, 1994.

11. C. Froese Fischer, Large-Scale Relativistic Calculations of Atomic Properties, European Research Conference on Relativistic Effects in Heavy Element Chemistry and Physics, Castelvecchio Pascoli, Italy (March 30 - April 4, 1995).

12. A. Aboussaï, M. Godefroid, T. Brage, C. Froese Fischer and P. Jönsson, MCHF calculations of hyperfine-induced transitions in the Breit-Pauli approximation, European Research Conference on Relativistic Effects in Heavy-Element Chemistry and Physics - Relativistic Quantum theory of Many-Electron Systems, (Castelvecchio Pascoli, Italy, 30 March - 4 April 1995) 
13. J. Carlsson, P. Jönsson, C. Froese Fischer and M. Godefroid, Large-scale MCHF calculations of isotope shifts and hyperfine structures in light atoms, 5th European Physical Society Conference on Atomic and Molecular Physics, (Edinburgh, Great Britan 3-7 April, 1995)

14. A. Aboussaïd, M. Godefroid, T. Brage and P. Jönsson, Hyperfine quenching of $1 s^{2} 2 s 2 p^{3} P_{0}^{o}$ in $C$ III and N IV, 5th European Physical Society Conference on Atomic and Molecular Physics, (Edinburgh, Great Britan 3-7 April, 1995)

15. C. Froese Fischer, P. Jönsson, A. Ynnerman, M. Godefroid, and J. Olsen, Large-scale MCHF calculations for ther Resonance Transition in NA: Hyperfine constants and Transition Probabilities, DAMOP Meeting (Toronto, May, 1995).

16. C. Froese Fischer, M. Godefroid and J. Olsen. MCHF calculations for $2 s^{2}{ }^{1} S-2 s 3 p{ }^{1} P$ transitions in some Be-like systems, 5 th International Colloquium on Atomic Spectra and Oscillator Strengths for Astrophysical and Laboratory Plasmas, (Paris, France 28-31 August, 1995)

17. P. Jönsson, A. Ynnerman, C. Froese Fischer, M. Godefroid and J. Olsen, Large-scale MCHF and $C I$ calculations of the transition probability and hyperfine structures in the sodium resonance transition, 5th International Colloquium on Atomic Spectra and Oscillator Strengths for Astrophysical and Laboratory Plasmas, (Paris, France 28-31 August, 1995)

18. P. Jönsson, C. Froese Fischer, M. Godefroid and J. Carlsson, Accurate calculations of isotope shifts and hyperfine structures in light atoms, 5 th International Colloquium on Atomic Spectra and Oscillator Strengths for Astrophysical and Laboratory Plasmas, (Paris, France 28-31 August, 1995)

19. M. Godefroid, P. Jönsson and C. Froese Fischer, Accurate transition probabilities for light atoms, 5th International Colloquium on Atomic Spectra and Oscillator Strengths for Astrophysical and Laboratory Plasmas, (Paris, France 28-31 August, 1995)

20. A. Abousaï, T. Brage, C. Froese Fischer, P.G. Judge, M. Godefroid and P. Jönsson, Hyperfine quenching of the $1 s^{2} 2 s 2 p{ }^{3} P_{0}$ state in $C I I I$ and $N I V$ and their astrophysical interest, 5th International Colloquium on Atomic Spectra and Oscillator Strengths for Astrophysical and Laboratory Plasmas, (Paris, France 28-31 August, 1995)

21. P. Jönsson and C. Froese Fischer, Convergence studies of atomic properties from variational methods: Transition energies, isotope shift, and hyperfine structure for the $1 s^{2} 2 s{ }^{2} S-$ $1 s^{2} 2 p^{2} P$ transitions in the $\mathrm{Li}$ isoelectronic sequence, DAMOP, May 14-18, 1996, Ann Arbor, MI

22. S. Fritzsche and C. Froese Fischer, Transition probabilities and lifetimes for phosphorus-like ions of the iron group, Zeeman International Conference on Atomic Physics, Amsterdam, Aug. 5-9, 1996.

\section{In Press or accepted}

1. G. Gaigalas and C. Froese Fischer, Extensions of the Hartree-Fock program to partially filled f-subshells, Comput. Phys. Commun. (accepted)

2. G. Godefroid, C. Froese Fischer, and P. Jönsson, Multiconfiguration Hartree-Fock calculations of atomic properties in light atoms, Physica Scripta (in press). 
3. S. Fritzsche and C. Froese Fischer, Radiative transition probabilities from non-orthogonal one-electron orbitals, Comput. Phys, Commun. (submitted).

4. C. Froese Fischer, M. Godefroid, and J. Olsen, Systematic Studies of the $2 s^{2}{ }^{1} S_{0}-2 s 3 p{ }^{1} P_{1}^{\circ}$ transition in the Be-like Sequence, J. Phys. B (submitted) 